\title{
Prevalence of infarct and villous clumps, and the expression of $\alpha$-smooth muscle actin in the placental basal plate in severe preeclampsia
}

\author{
MIN KONG ${ }^{1,2}$, LIANLIAN LIU ${ }^{1}$, FEI YI $^{3}$, MUHAMMAD KHAN $^{3}$, \\ AZHAR RASUL ${ }^{3}$, FAN YANG ${ }^{1}$, XIAOYAN MA ${ }^{1}$ and TONGHUI MA ${ }^{3}$ \\ ${ }^{1}$ Department of Obstetrics and Gynecology, Jilin University Bethune Second Hospital, Changchun, \\ Jilin 130041; ${ }^{2}$ Department of Obstetrics and Gynecology, First People's Hospital of Jining, Jining, Shandong 272111; \\ ${ }^{3}$ Central Research Laboratory, Jilin University Bethune Second Hospital, Changchun, Jilin 130041, P.R. China
}

Received February 14, 2013; Accepted July 8, 2013

DOI: $10.3892 / \mathrm{mmr} .2013 .1651$

\begin{abstract}
The present study was conducted to investigate the prevalence of multifocal infarcts and multifocal villous clumps, as well as the expression of $\alpha$-smooth muscle actin ( $\alpha$-SMA) in the basal plate of the placenta. Multifocal infarcts and/or multifocal villous clumps were more frequently observed in early onset severe preeclampsia (EOSPE; $n=20$ ) compared with the early control $(\mathrm{n}=18)$, late onset severe preeclampsia (LOSPE; $\mathrm{n}=20)$ and late control $(\mathrm{n}=20)$ groups. Infarct in transitional and late stages was significantly more frequent compared with that in the early stage in EOSPE. In LOSPE, the infarct in the early stage was significantly increased compared with transitional and late stages. The expression of $\alpha$-SMA mRNA and protein was determined by RT-PCR and western blotting, respectively, and increased progressively in late, transitional and early infarct, and positively correlated with the number of smooth muscle cells of villous vessels and myofibroblasts of villous stroma studied by immunohistochemistry. These results indicated that multifocal infarct and multifocal villous clumps may affect the blood flow through the basal plate, not only by directly blocking vessels, but also by indirectly making the vessels extruded by contraction of cells stained by $\alpha$-SMA In addition, foci in the early stage may be more important in affecting the contractility of the placental basal plate.
\end{abstract}

\section{Introduction}

Preeclampsia (PE) is a severe medical syndrome affecting pregnant females. $\mathrm{PE}$ is characterized by new onset hyperten-

Correspondence to: Professor Xiaoyan Ma, Department of Obstetrics and Gynecology, Jilin University Bethune Second Hospital, 218 Ziqiang Street, Changchun, Jilin 130041, P.R. China E-mail: mxytheblessed@sina.com

Key words: severe preeclampsia, $\alpha$-smooth muscle actin, infarct, villous clump, basal plate sion and proteinuria following 20 weeks of pregnancy, and threatens maternal and child health, as well as survival (1). The condition is subclassified into early and late onset according to standards previously outlined by von Dadelszen et al (2). However, the pathogenesis of PE is unclear. Previously, a number of studies have focused on the pathological changes involved in PE, including infarct, fibrosis, arteriosclerosis and decidual arterioles of the basal plate of the placenta, that affect perfusion (3-5). However, these changes are not marked in the basal plate of specific severe PE (SPE) patients, particularly in the placenta of late onset SPE (LOSPE) and the SPE accompanied by diabetes mellitus and multiple pregnancies (6).

The basal plate of the placenta is an important region which controls nutrient-rich and oxygen-rich maternal blood flowing into the intervillous space. It also regulates metabolic products and carbon dioxide-rich fetal blood which flows from the intervillous space into the maternal circulation (7). Pathological changes, including fibrosis, arteriosclerosis and decidual arterioles, may cause the obstruction of the vessels in the basal plate resulting in metabolic accumulation and placental underperfusion, which may induce hypoxia, oxidative stress and inflammation of the placenta (8). These changes may also lead to maternal vascular endothelial dysfunction and clinical manifestations, such as hypertension and proteinuria (1).

It is well-known that extravillous trophoblasts (EVTs) invade into the spiral arteries of the decidua and superficial myometrium to establish flaccid low-resistance arteries during pregnancy (9). If the EVTs fail to remold the spiral arteries in the decidua and superficial myometrium, the resistance of vessels in the basal plate may increase and cause the pathogenesis of SPE (10). Previous studies on the pathological changes of the placenta have also revealed that increased contraction components exist in the regions of EVT shallow implantation in the placental bed $(11,12) . \alpha$-smooth muscle actin $(\alpha$-SMA) is a component of microfilaments and contributes to the cytoskeleton in specific contractile cells, including smooth muscle cells (SMCs) and myofibroblasts of the villous stroma in the basal plate (13).

To date, it has been hypothesized that specific anchoring villi contain SMCs and villous stromal cells but not free EVTs 
in the normomorph (normal structure) basal plate. In addition, it has been reported that specific anchoring villi are involved in infarct foci and/or other regions, which may contain cells with contractile function $(9,10)$. Hence, we hypothesized that expression of $\alpha$-SMA may be increased in an elevated number of potentially contractile cells in the basal plate with pathological changes, inducing the pathogenic process of SPE. However, few studies have analyzed these pathological changes in the non-infarct area of the villous clump. Therefore, the present study was designed to explore the expression profile of $\alpha$-SMA in the foci of infarcts and villous clumps in the basal plate to determine the correlation between $\alpha$-SMA and SPE. An additional aim was to identify the main placental pathological changes of patients with LOSPE.

\section{Materials and methods}

Samples collection. This study was approved by the ethics committee of Jilin University Bethune Second Hospital and all subjects provided written informed consent. Females with normal or preeclamptic pregnancies were defined according to criteria previously outlined by Zhao et al (14). A total of 78 placentas (38 from normal and 40 from preeclampsia) were used in this study. The patient demographic data are summarized in Table I.

Tissues explants. Tissues from the basal plate were selected with infarct, villous clumps and normomorph. Infarct samples represented three periods: early (purplish and soft), transitional (yellow and stiff) and late (white and stiff) infarct (15). Villous clump is tissue that is a little thicker and whiter than normomorph. All the tissues were collected within 5 min after the placenta was delivered. One section was stored at $-80^{\circ} \mathrm{C}$ to prepare for the extraction of $\mathrm{mRNA}$ and protein, and the other was fixed in $4 \%$ formaldehyde to prepare the sample for immunohistochemistry.

Immunohistochemistry. Placental tissues were fixed in $4 \%$ formaldehyde and then embedded into paraffin blocks. After deparaffinizing in xylene and dehydrating in a gradient ethanol, the $2.5-\mu \mathrm{m}$ thick sections were heated for $20 \mathrm{~min}$ in the microwave to repair antigens. Next, $3 \%$ hydrogen peroxide was used to quench the activity of endogenous peroxidase at room temperature for $10 \mathrm{~min}$. The slides were incubated with $\alpha$-SMA monoclonal antibody obtained from mouse (1:200; Zhongshan Golden Bridge Biotechnology Co., Ltd, Beijing, China) for $60 \mathrm{~min}$ at $37^{\circ} \mathrm{C}$ in a humidified chamber. Poly-HRP anti-mouse/rabbit IgG (PV-9000 2-step plus, Zhongshan Golden Bridge Biotechnology Co., Ltd) was added to the slides for $50 \mathrm{~min}$ at $37^{\circ} \mathrm{C}$ in a humidified chamber. Diaminobenzidine kit (Zhongshan Golden Bridge Biotechnology Co., Ltd) was used to detect $\alpha$-SMA staining in the basal plate. Following counterstaining by hematoxylin, redehydrating by graded ethanol and vitrification by dimethylbenzene, the slides were mounted in neutral balsam. The primary antibody was replaced by PBS for $\alpha$-SMA-negative control. The vessels in the placental parenchyma were used for a positive control. The slides were assessed by light microscope (Bx51; Olympus Corporation, Japan) by two independent pathologists. The brown cytoplasm and membrane
Table I. Demographic data of patients in all groups.

\begin{tabular}{lccc}
\hline Group & Cases, $n$ & Age, years & $\begin{array}{c}\text { Pregnancy } \\
\text { duration, weeks }\end{array}$ \\
\hline EOSPE & 20 & $28.66 \pm 6.33$ & $28.86 \pm 3.64$ \\
LOSPE & 20 & $29.05 \pm 7.01$ & $37.63 \pm 1.49$ \\
EC & 18 & $25.00 \pm 6.89$ & $28.23 \pm 3.45$ \\
LC & 20 & $28.20 \pm 5.75$ & $37.44 \pm 1.56$ \\
\hline
\end{tabular}

EOSPE, early onset severe preeclampsia; LOSPE, late onset severe preeclampsia; EC, early control; LC, late control.

of SMCs and stromal cells stained by $\alpha$-SMA antibody were analyzed positively.

RNA preparation and semi-quantitative $R T-P C R$. Total RNA was extracted from the samples by TRIzol extraction (Invitrogen Life Technologies, Carlsbad, CA, USA). RNA concentration and purity was determined by absorbance at 260 and $280 \mathrm{~nm}\left(\mathrm{OD}_{260 / 280},>1.8\right.$ and $\left.<2.1\right)$ using a spectrophotometer (NanoDrop 2000; NanoDrop products, Wilmington, DE, USA). RT-PCR (BioRT Two Step RT-PCR, China) was performed by converting $1 \mu \mathrm{g}$ RNA into cDNA. Reverse transcription was performed according to the manufacturer's instructions. The reaction system of cDNA systhesis had a total volume of $10 \mu \mathrm{l}$, consisting of $5 \mathrm{X}$ RT buffer, dNTP mixture (10 mM), oligo-dT, RNase-inhibitor, AMV reverse transcriptase, RNA sample and RNase free $\mathrm{H}_{2} \mathrm{O}$. The total PCR volume was $25 \mu \mathrm{l}$, containing: 10X PCR buffer, dNTP mixture (10 mM), primer, Taq mix DNA polymerase, cDNA and double distilled $\mathrm{H}_{2} \mathrm{O}$. The amplification of nucleic acids was performed using 30 cycles of denaturation at $94^{\circ} \mathrm{C}$ for $30 \mathrm{sec}$, annealing at $58^{\circ} \mathrm{C}$ for $30 \mathrm{sec}$, extension at $72^{\circ} \mathrm{C}$ for $1 \mathrm{~min}$ and re-extension at $72^{\circ} \mathrm{C}$ for $5 \mathrm{~min}$. The primers for $\alpha$-SMA were (forward) 5'-GCGTGGCTATTCCTTCGTTAC-3' and (reverse) 5'-CATAGTGGTGCCCCCTGATAG-3', amplified to a 331-bp fragment. The housekeeping gene, GAPDH, served as an internal control. The primers for GAPDH were (forward) 5'-GAAGGTGAAGGTCGGAGT-3' and (reverse) 5'-GAAGATGGTGATGGGATTTC-3', amplified to a 226-bp fragment. Primers were designed by Primer software and synthesized by Sangon (Shanghai, China). PCR products were subjected to gel electrophoresis in $1.5 \%$ agarose (Invitrogen Life Technologies) followed by staining using bromophenol and were detected by MultiImage Light Cabinet filter positions (Alpha Innotech, San Leandro, CA, USA) and analyzed by Image J software.

Protein isolation and western blotting. Basal plate tissues were homogenized in lysis buffer [50 mM Tris- $\mathrm{HCl}(\mathrm{pH} 8.0)$, $150 \mathrm{mM} \mathrm{NaCl}, 1 \%$ Triton-X100, 0.5\% NaDC, $1 \%$ NP-40 and $1 \% \mathrm{SDS}]$ on ice and were centrifuged at 9,180 x g for $8 \mathrm{~min}$ at $40^{\circ} \mathrm{C}$. Western blotting was performed as described previously $(16,17)$. Membranes were immunoblotted by mouse monoclonal $\alpha$-SMA antibody (1:1,000; Beyotime Institute of Biotechnology, Shanghai, China) overnight at $4^{\circ} \mathrm{C}$. Membranes were then washed three times with TBS-T and incubated 
with peroxidase-conjugated Affinipure goat anti-mouse IgG (1:1,000; Zhongshan Golden Bridge Biotechnology Co., Ltd) at room temperature for $60 \mathrm{~min}$. Expression of $\alpha$-SMA protein was detected using an enhanced chemiluminescence system (Millipore, Billerica, MA, USA) and was exposed on film (Eastman Kodak Company, Rochester, NY, USA). GAPDH expression was used as the control.

Statistical analysis. SPSS 17.0 software was used to analyze the data (SPSS, Inc., Chicago, IL, USA). The data obtained obey the normal distribution presented as the mean \pm SD. One-way ANOVA was applied and significant differences were observed between the groups. $\chi^{2}$ test was used to provide evidence whether the prevalence among all the groups was significantly different. $\mathrm{P}<0.05$ was considered to indicate a statistically significant difference. Semiquantitative RT-PCR and western blotting were performed in triplicate.

\section{Results}

Prevalence of multifocal infarct and villous clumps in all groups. Firstly, the prevalence of multifocal infarct and villous clumps in all groups was determined and results revealed that the prevalence of multifocal infarct was $90.00 \%$ in 20 EOSPE patients, $15.00 \%$ in 20 LOSPE patients, $5.56 \%$ in $18 \mathrm{EC}$ patients and $10.00 \%$ in 20 LC patients, respectively $\left(\chi^{2}=44.39 ; \mathrm{P}<0.05\right)$. The prevalence of villous clumps was EOSPE $>$ LOSPE $>$ LC $>$ EC (75.00, 60.00, 35.00 and $11.11 \%$, respectively; $\chi^{2}=18.14$; $\mathrm{P}<0.05$; Table II).

The prevalence of multifocal infarct in EOSPE was markedly higher than EC $(\mathrm{P}=0.000)$ and LOSPE $(\mathrm{P}=0.000)$. In addition, the prevalence of multifocal villous clumps was significantly higher in EOSPE compared with EC $(\mathrm{P}=0.000)$ and LOSPE compared with LC ( $\mathrm{P}=0.003$; Table II).

Localization of $\alpha$-SMA in the basal plate. It is well established that the placenta plays a key role in the pathogenesis of SPE. To understand the pathological changes in SPE placenta, placentas obtained from normal pregnancy and SPE females were compared. To examine the localization of $\alpha$-SMA in various regions of the basal plate, including the infarct (early, transitional and late infarct area), villous clumps and normal areas, anti- $\alpha$-SMA immunohistochemistry was performed. Immunohistochemical staining results showed that $\alpha$-SMA was expressed in the placenta of all groups and was largely localized to the SMCs of villous vessels and myofibroblasts of the villous stroma in the basal plate. In addition, $\alpha$-SMA was observed in the cytomembrane and cytoplasm of these contractile cells under the microscope (Fig. 1).

Identification of villi in infarct and villous clumps. To characterize the morphological changes in various regions of the basal plate, villi in infarct and villous clumps were observed. $\alpha$-SMA staining demonstrated that the morphology of villi varied in early, transitional and late infarct. Typical early infarct of the basal plate exhibited characteristics of collapsed intervillous space. Features of denaturation and necrosis of trophoblast cells and ghost villi were observed in the typical transitional infarct. Late infarct is the final stage of infarct and exhibits the characteristics of extensive fibrosis. The basal plate tissues
Table II. Prevalence of multifocal infarct and villous clumps in the basal plate in all groups.

\begin{tabular}{lccc}
\hline Group & Cases, $\mathrm{n}$ & $\begin{array}{c}\text { Multifocal } \\
\text { infarct, } \mathrm{n}(\%)\end{array}$ & $\begin{array}{c}\text { Multifocal } \\
\text { villous clump, } \mathrm{n}(\%)\end{array}$ \\
\hline EOSPE & 20 & $18(90.00)$ & $15(75.00)$ \\
LOSPE & 20 & $3(15.00)$ & $12(60.00)$ \\
EC & 18 & $1(5.56)$ & $2(11.11)$ \\
LC & 20 & $2(10.00)$ & $3(35.00)$ \\
\hline
\end{tabular}

EOSPE, early onset severe preeclampsia; LOSPE, late onset severe preeclampsia; EC, early control; LC, late control.

appeared to be a little thicker and whiter than normomorph tissues. However, under the microscope, different numbers, sizes and stages of villous clumps were observed (Fig. 1).

Proportion of infarct and villous clumps of various stages in EOSPE and LOSPE. As stated, histological observations of the infarct and villous clumps usually varied based on the stages under the microscope, we moved forward a single step to make it clear that the proportion of early, transitional and late stage in multifocal infarct and multifocal villous clump. Results showed that infarct in transitional and late stages was markedly more frequent than that in early stage in EOSPE. In LOSPE, the infarct in the early stage was significantly increased compared with transitional and late stages (Table III). The area of multifocal villous clump showed the same trend with multifocal infarct.

Expression levels of $\alpha$-SMA in multifocal infarct. To determine the expression levels of $\alpha$-SMA in multifocal infarct and villous clumps, mean density of $\alpha$-SMA was determined in multifocal infarct in early, transitional and late stages. Results demonstrate that the expression levels of $\alpha$-SMA were lowest in the normomorph, followed by late, transitional and early infarct in all groups $(\mathrm{P}<0.05$; Fig. 2).

Correlation between expression levels of $\alpha$-SMA and number of cells stained in basal plate infarcts. To investigate the correlation between expression levels of $\alpha$-SMA and the number of stained cells, integrated optical density values in normomorph and late, transitional and early multifocal infarct samples were obtained and stained cells were quantified. The results revealed that $\alpha$-SMA expression levels increased in normomorph, late, transitional and early multifocal infarct progressively $(\mathrm{P}<0.05)$, and positively correlated with the number of stained cells in the basal plate $(\mathrm{r}=0.48, \mathrm{P}=0.07$; $\mathrm{r}=0.652, \mathrm{P}=0.000 ; \mathrm{r}=0.544, \mathrm{P}=0.002$; and $\mathrm{r}=0.472, \mathrm{P}=0.008$, respectively; Fig. 3).

Expression of $\alpha-S M A m R N A$ in basal plate infarcts. Immunohistochemical results demonstrated that the expression levels of $\alpha$-SMA were highest in early infarct, followed by transitional infarct, late infarct and normomorph. To determine the expression levels of $\alpha$-SMA mRNA, RT-PCR was performed. Results of RT-PCR showed that the expression levels of $\alpha$-SMA 

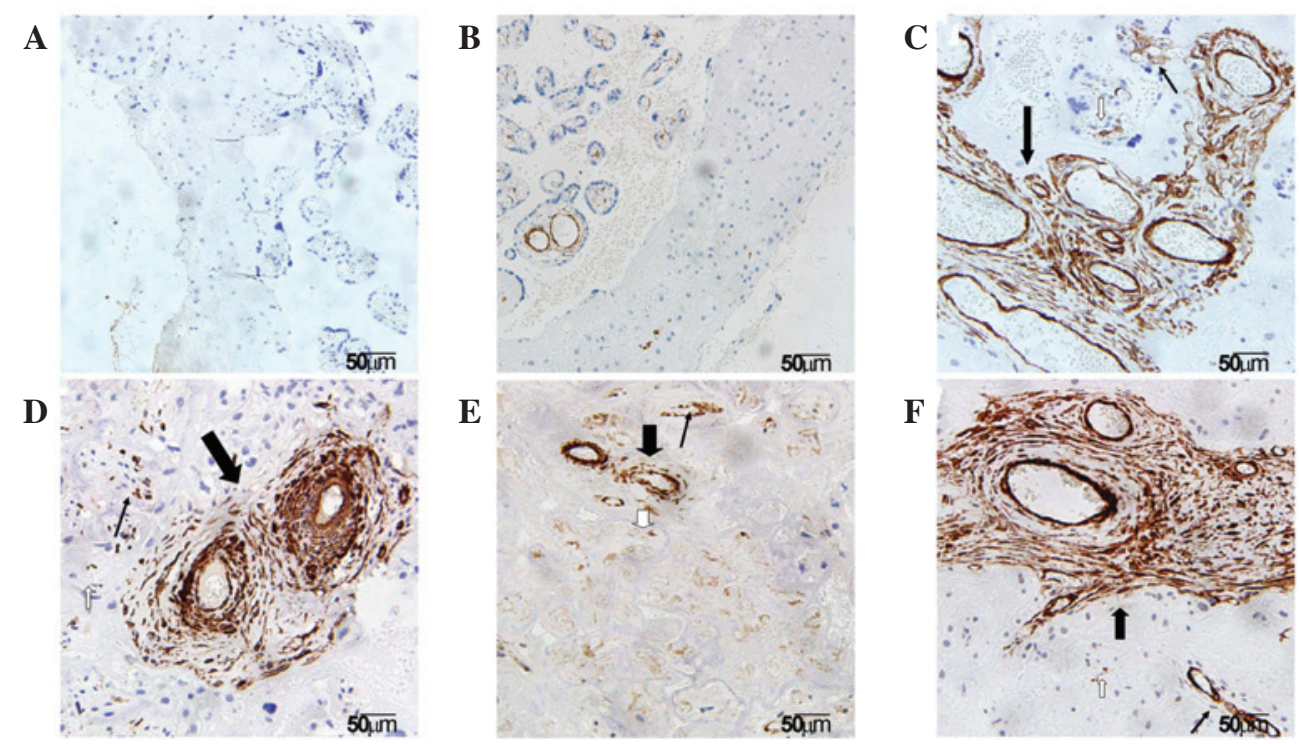

Figure 1. Immunohistochemical detection of $\alpha$-SMA in basal plate tissues. (A) Blank control, (B) parenchyma, (C) early infarct, (D) transitional infarct, (E) late infarct and (F) villous clumps. Solid arrow, big villus; hollow arrow, medium villus; small arrow, small villus. Scale bar, $50 \mu$ m. $\alpha$-SMA, $\alpha$-smooth muscle actin.

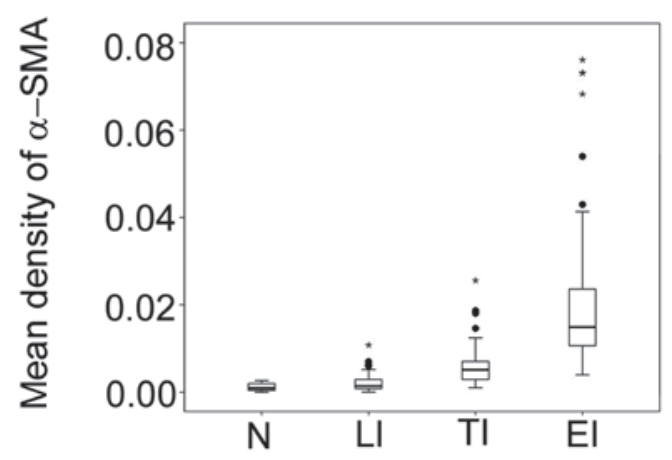

Figure 2. Mean density of $\alpha$-SMA in basal plate infarct. N, normomorph; LI, late infarct; TI, transitional infarct; EI, early infarct; $\alpha$-SMA, $\alpha$-smooth muscle actin.

mRNA increased in normomorph, late, transitional and early infarct samples progressively. The $\alpha$-SMA mRNA expression levels were determined by optical density (Fig. 4) and the results were consistent with immunohistochemical results.

Expression of $\alpha$-SMA protein in basal plate infarcts. As our previous results showed that $\alpha$-SMA mRNA was upregulated in basal plate infarcts, $\alpha$-SMA protein levels were analyzed in SPE patients by western blotting. Expression levels of $\alpha$-SMA protein were progressively higher in early, transitional and late infarct samples compared with normomorph placental tissues. In addition, levels in early infarct were higher than late infarct and transitional infarct among the SPE patients while levels in transitional infarct were higher than late infarct (Fig. 5). These results indicate that, in addition to $\alpha$-SMA mRNA levels, $\alpha$-SMA proteins levels are also altered in SPE patients.

\section{Discussion}

SPE is a complication of pregnancy associated with a number of organ systems, including renal, retinal, cerebral and cardio- vascular, and correlates with significant maternal and fetal morbidity and mortality worldwide (18). It is generally accepted that the placenta is a causative organ to SPE patients and when it is removed from the uterus, clinical manifestations, including hypertension and proteinuria are easily controlled (19).

The main hypothesis of shallow implantation of the placenta was the abnormal villous cytotrophoblast caused by immune factors, genetic factors and so on, invading into the spiral arteries of maternal decidua and myometrium, which failed to establish flaccid low-resistance uteroplacental arteries (20). Remodeling of uterine spiral arteries is important for the growth and development of the fetus. The failure of arterial remolding is associated with relatively hypoxic trophoblast tissue and a state of oxidative stress in the placenta. The hypoxia/oxidative stress may then result in clinical manifestation of SPE, including hypertension and proteinuria. However, this hypothesis is based on previous observations in which the basal plate was misidentified as the placental bed (21). As it is difficult to collect specimens from the maternal decidua and myometrium of SPE patients and the control group (22), these results do not provide convincing evidence to confirm that shallow implantation is responsible for the pathogenesis of SPE.

Infarct foci and villous clumps may lead to malperfusion and hypoxia of the placenta, and the dysfunction of villi involved in these areas. To date, multifocal infarct, as well as multifocal infarct of the placental basal plate, has not been described. The term multifocal infarct of the placental basal plate was defined by reference to the definition of pervasive infarct of the placenta (23). The multifocal infarct of the placental basal plate is the infarct area covering $15 \%$ of the total placental basal plate. It has been widely accepted that pathological changes in the basal plate infarct may cause underperfusion of the placenta in EOSPE with or without fetal growth restriction (24). However, few studies have analyzed the microchanges of placental pathology affecting placental perfusion in the non-infarct area. In the present study, villous 
Table III. Proportion of infarct in various stages in SPE.

\begin{tabular}{lcccc}
\hline Group & Cases, $\mathrm{n}$ & Multifocal infarct, $\mathrm{n}(\%)$ & Early infarct, $\mathrm{n}(\%)$ & Transitional-late infarct, $\mathrm{n}(\%)$ \\
\hline EOSPE & 20 & $18(90.00)$ & $6(33.33)$ & $12(66.67)$ \\
LOSPE & 20 & $3(15.00)$ & $2(10.00)$ & $1(5.00)$
\end{tabular}

EOSPE, early onset severe preeclampsia; LOSPE, late onset severe preeclampsia; SPE, severe preeclampsia.

A

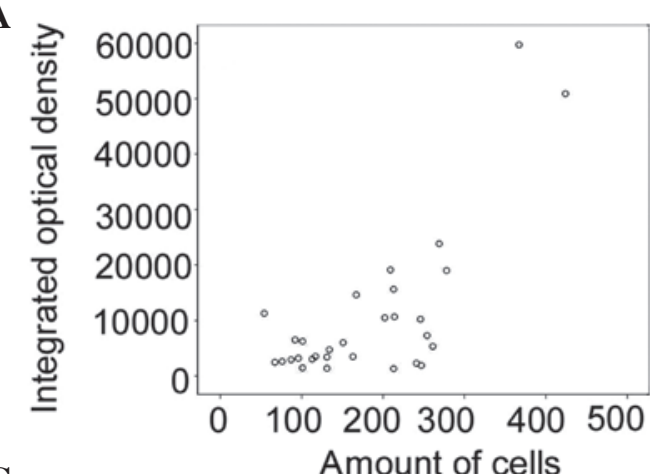

C

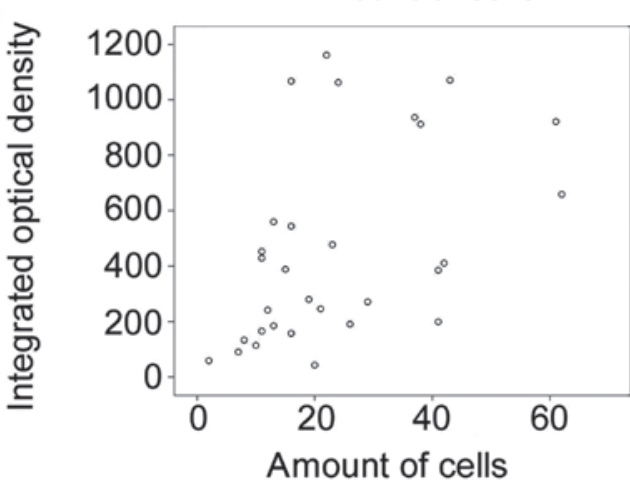

B

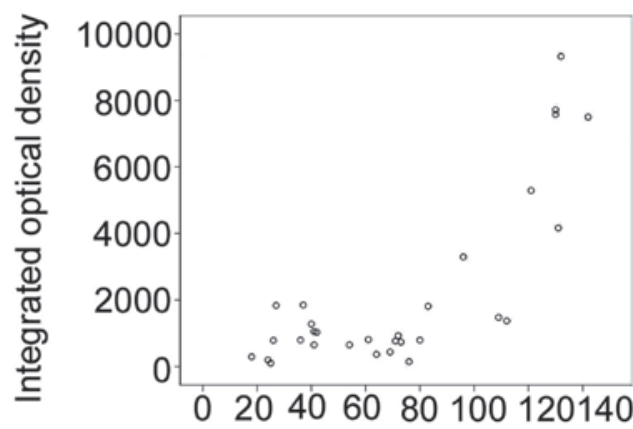

D

Amount of cells

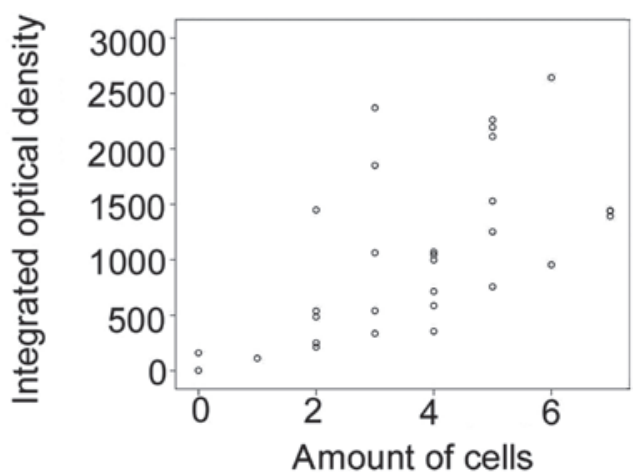

Figure 3. Correlation between integrated optical density and amounts of cells stained in basal plate infarcts. (A) Early infarct, (B) transitional infarct, (C) late infarct and (D) normomorph.

\section{L1}
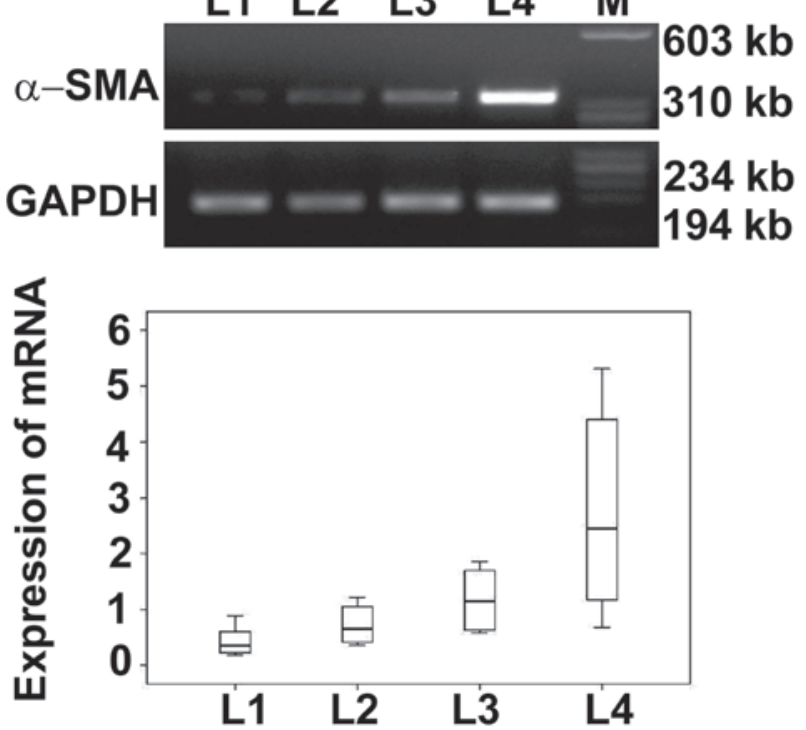

Figure 4. Expression of $\alpha$-SMA mRNA in basal plate infarcts. The expression of $\alpha$-SMA mRNA was detected at $331 \mathrm{bp}$ as a single band. GAPDH served as internal control. M, marker; L1, normomorph; L2, late infarct; L3 transitional infarct; L4, early infarct; $\alpha$-SMA, $\alpha$-smooth muscle actin.
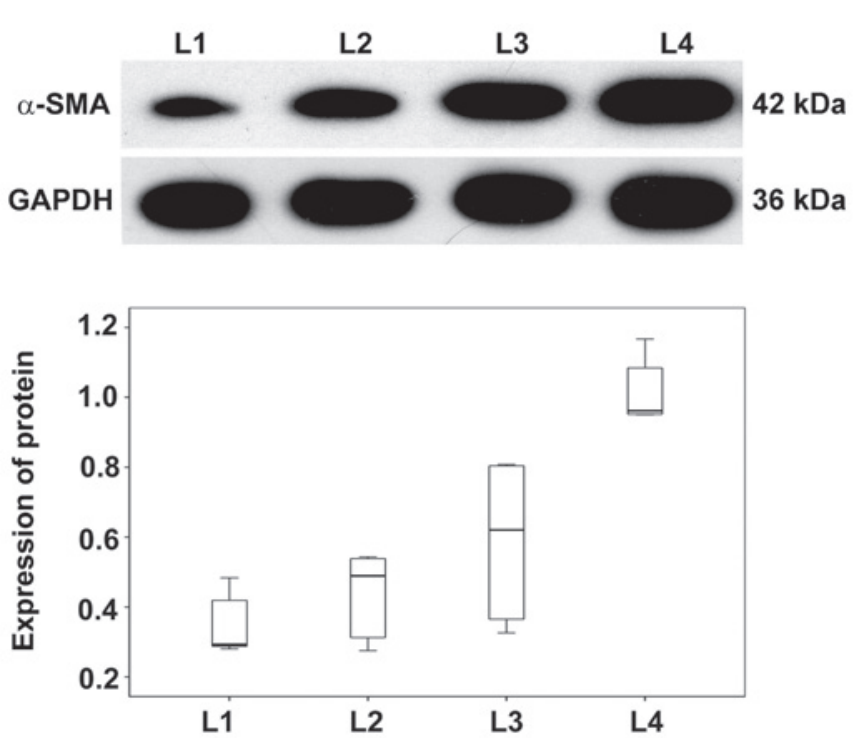

Figure 5. Expression of $\alpha$-SMA protein in basal plate infarcts. L1, normomorph; L2, late infarct; L3, transitional infarct; L4, early infarct; $\alpha$-SMA, $\alpha$-smooth muscle actin. 
clumps exhibiting a normomorph appearance were observed in the basal plate without infarct foci with the naked eye. Similarly, the multifocal villous clumps of the basal plate was the area of villous clump which covered $15 \%$ of the total basal plate. In various prevalences between these groups, the multifocal infarct of the basal plate was the main pathological change of LOSPE, while multifocal villous clumps of the basal plate was the common pathway in the pathogenic process of EOSPE and LOSPE. In addition, these observations are not consistent with the traditional hypothesis that placental lesions associated with maternal underperfusion are less frequently observed in LOSPE than in EOSPE (24).

The increased number of contractile proteins in the basal plate may represent an additional pathological change which affects placental perfusion and aggravates uterine tension. To date, few studies have discussed this hypothesis. Actin is a cytoskeleton protein with contractile function and has at least six distinct isoforms in the vertebrate tissues (25). The actin proteins include $\alpha$-skeletal muscle actin, $\alpha$-cardiac muscle actin, $\alpha$-smooth muscle actin, $\beta$-actin, $\gamma$-smooth muscle actin and $\gamma$-nonmuscle actin (26). Matsumura et al (13) reported that the human placenta expresses three isoforms of actin, $\beta$-actin, $\alpha$-SMA and $\gamma$-SMA, which account for 60,30 and $10 \%$, respectively. $\beta$-actin largely localizes within the extravascular stroma while $\alpha$-SMA localizes in endovascular tissues. In addition, $\alpha$-SMA is a biomarker of myofibroblasts (27). Results of the present study indicate that $\alpha$-SMA is largely localized to the cytomembrane and cytoplasm of the SMCs of villi and myofibroblasts of villous stroma in the basal plate. These villi mainly spread over infarct foci and villous clumps. These results are consistent with previous studies indicating that $\alpha$-SMA is expressed in various cells, including mesenchyme, reticulum, smooth muscle, hofbauer, filamented and vacuolated cells, and fibroblasts and myofibroblasts in the stroma (28). The villi components labeled by $\alpha$-SMA mainly exist in infarcts and villous clumps of the basal plate. Current staining results indicate that the contraction of these $\alpha$-SMA-labeled cells may play a role in the pathogenesis of SPE by affecting maternal circulation and inducing the contractility of uterine.

Tannheimer et al (29) previously showed that myofibroblasts participate in injury repair by secreting collagen, extracellular matrix and proinflammatory mediators. During oxidative stress, the structure of actin may be damaged and the cells stained by $\alpha$-SMA may be undergoing cytoclasis and fibrosis (30). These concepts provide a good explanation for the altered expression profile of $\alpha$-SMA in normomorph, late, transitional and early infarct samples in the basal plate. The expression and contraction of $\alpha$-SMA may vary in a time-dependent manner and a longer time under conditions of hypoxia, and a higher rate of cell degeneration and fibrosis enables lower expression of $\alpha$-SMA and weaker contraction of $\alpha$-SMA.

More importantly, results of the present study may be useful for clinical application. To date, the only method to control SPE is termination of pregnancy, which may increase the rate of preterm births and neonatal mortalities of SPE. The early infarct and villous clumps of basal plate may be the target of minimally invasive interventional treatment through ultrasonic Doppler.

In conclusion, the present study shows that multifocal infarcts and multifocal villous clumps may participate in the pathogenic progress of SPE by blocking blood vessels, inducing vessel contraction and aggravating uterine tension by $\alpha$-SMA.

\section{Acknowledgements}

This study was funded by grants from the Science and Technology Department of Jilin Province (no. 20090464) and the Science and Technology Agency of Changchun (no. 08SF44). The authors would like to thank Mei Sun and Yang Xia (Department of Pathology, Jilin University Bethune Second Hospital) for their assistance in observing placental pathology.

\section{References}

1. George EM and Granger JP: Recent insights into the pathophysiology of preeclampsia. Expert Rev Obstet Gynecol 5: 557-566, 2010.

2. von Dadelszen P, Magee LA and Roberts JM: Subclassification of preeclampsia. Hypertens Pregnancy 22: 143-148, 2003.

3. Hargitai B, Marton T and Cox PM: Best practice no 178 Examination of the human placenta. J Clin Pathol 57: 785-792, 2004.

4. Khong TY and Werger AC: Myometrial fibers in the placental basal plate can confirm but do not necessarily indicate clinical placenta accreta. Am J Clin Pathol 116: 703-708, 2001.

5. Vogler C, Petterchak J, Sotelo-Avila C and Thorpe C: Placental pathology for the surgical pathologist. Adv Anat Pathol 7: 214-229, 2000.

6. Ramma W and Ahmed A: Is inflammation the cause of pre-eclampsia? Biochem Soc Trans 39: 1619-1627, 2011.

7. von Versen-Hoeynck FM and Powers RW: Maternal-fetal metabolism in normal pregnancy and preeclampsia. Front Biosci 12: 2457-2470, 2000.

8. Lash GE, McLaughlin BE, MacDonald-Goodfellow SK, et al: Relationship between tissue damage and heme oxygenase expression in chorionic villi of term human placenta. Am J Physiol Heart Circ Physiol 284: H160-H167, 2003.

9. Myatt L: Role of placenta in preeclampsia. Endocrine 19: 103-111, 2002.

10. Whitley GS and Cartwright JE: Cellular and molecular regulation of spiral artery remodelling: lessons from the cardiovascular field. Placenta 31: 465-474, 2010.

11. Furuya M, Ishida J, Aoki I and Fukamizu A: Pathophysiology of placentation abnormalities in pregnancy-induced hypertension. Vasc Health Risk Manag 4: 1301-1313, 2008.

12. Lyall F: Priming and remodelling of human placental bed spiral arteries during pregnancy - a review. Placenta 26 (Suppl A): S31-S36, 2005.

13. Matsumura S, Sakurai K, Shinomiya T, Fujitani N, Key K and Ohashi M: Biochemical and immunohistochemical characterization of the isoforms of myosin and actin in human placenta. Placenta 32: 347-355, 2011.

14. Zhao S, Gu Y, Fan R, Groome LJ, Cooper D and Wang Y: Proteases and sFlt-1 release in the human placenta. Placenta 31: 512-518, 2010.

15. Kaufmann P, Luckhardt M, Schweikhart G and Cantle SJ: Cross-sectional features and three-dimensional structure of human placental villi. Placenta 8: 235-247, 1987.

16. Rasul A, Yu B, Zhong L, Khan M, Yang H and Ma T: Cytotoxic effect of evodiamine in SGC-7901 human gastric adenocarcinoma cells via simultaneous induction of apoptosis and autophagy. Oncol Rep 27: 1481-1487, 2012.

17. Rasul A, Yu B, Khan M, Zhang K, Iqbal F, Ma T and Yang H: Magnolol, a natural compound, induces apoptosis of SGC-7901 human gastric adenocarcinoma cells via the mitochondrial and PI3K/Akt signaling pathways. Int J Oncol 40: 1153-1161, 2012.

18. Paruk F and Moodley J: Maternal and neonatal outcome in early- and late-onset pre-eclampsia. Semin Neonatol 5: 197-207, 2000.

19. Redman CW and Sargent IL: Placental debris, oxidative stress and pre-eclampsia. Placenta 21: 597-602, 2000.

20. Hung TH and Burton GJ: Hypoxia and reoxygenation: a possible mechanism for placental oxidative stress in preeclampsia. Taiwan J Obstet Gynecol 45: 189-200, 2006. 
21. Kaufmann P, Black S and Huppertz B: Endovascular trophoblast invasion: implications for the pathogenesis of intrauterine growth retardation and preeclampsia. Biol Reprod 69: 1-7, 2003.

22. Craven CM, Morgan T and Ward K: Decidual spiral artery remodelling begins before cellular interaction with cytotrophoblasts. Placenta 19: 241-252, 1998.

23. Crum CP, Nucci MR and Lee KR: Diagnostic Gynecologic and Obstetric Pathology. 2nd ed. Saunders/Elsevier, Philadelphia, PA, p1202, 2011.

24. Ogge G, Chaiworapongsa T, Romero R, et al: Placental lesions associated with maternal underperfusion are more frequent in early-onset than in late-onset preeclampsia. J Perinat Med 39: 641-652, 2011

25. Vandekerckhove $\mathrm{J}$ and Weber $\mathrm{K}$ : At least six different actins are expressed in a higher mammal: an analysis based on the amino acid sequence of the amino-terminal tryptic peptide. J Mol Biol 126: 783-802, 1978.

26. Glukhova MA, Frid MG and Koteliansky VE: Developmental changes in expression of contractile and cytoskeletal proteins in human aortic smooth muscle. J Biol Chem 265: 13042-13046, 1990
27. Teraoka R, Shimada $\mathrm{T}$ and Aburada M: The molecular mechanisms of the hepatoprotective effect of gomisin A against oxidative stress and inflammatory response in rats with carbon tetrachloride-induced acute liver injury. Biol Pharm Bull 35: 171-177, 2012.

28. Sati L, Seval Y, Yasemin Demir A, Kosanke G, Kohnen G and Demir R: Cellular diversity of human placental stem villi: an ultrastructural and immunohistochemical study. Acta Histochem 109: 468-479, 2007.

29. Tannheimer SL, Wright CD and Salmon M: Combination of roflumilast with a beta-2 adrenergic receptor agonist inhibits proinflammatory and profibrotic mediator release from human lung fibroblasts. Respir Res 13: 28, 2012.

30. Rogers KR, Morris CJ and Blake DR: The cytoskeleton and its importance as a mediator of inflammation. Ann Rheum Dis 51: 565-571, 1992. 